\title{
Validity of diagnostic codes and laboratory measurements to identify patients with idiopathic acute liver injury in a hospital database
}

\author{
Renate Udo ${ }^{1,2}$, Anke H. Maitland-van der Zee ${ }^{1}$, Toine C. G. Egberts ${ }^{1,3}$, Johanna H. den Breeijen ${ }^{1,3}$, \\ Hubert G. M. Leufkens ${ }^{1,2}$, Wouter W. van Solinge ${ }^{1,4}$ and Marie L. De Bruin ${ }^{1,2 *}$ \\ ${ }^{1}$ Division of Pharmacoepidemiology and Clinical Pharmacology, Utrecht Institute for Pharmaceutical Sciences, Utrecht University, The \\ Netherlands \\ ${ }^{2}$ Medicines Evaluation Board, Utrecht, The Netherlands \\ ${ }^{3}$ Department of Clinical Pharmacy, University Medical Center Utrecht, The Netherlands \\ ${ }^{4}$ Department of Clinical Chemistry and Haematology, University Medical Center Utrecht, Utrecht, The Netherlands
}

\begin{abstract}
Purpose The development and validation of algorithms to identify cases of idiopathic acute liver injury (ALI) are essential to facilitate epidemiologic studies on drug-induced liver injury. The aim of this study is to determine the ability of diagnostic codes and laboratory measurements to identify idiopathic ALI cases.

Methods In this cross-sectional validation study, patients were selected from the hospital-based Utrecht Patient Oriented Database between 2008 and 2010. Patients were identified using (I) algorithms based on ICD-9-CM codes indicative of idiopathic ALI combined with sets of liver enzyme values (ALT $>2 \times$ upper limit of normal (ULN); AST $>1$ ULN + AP $>1$ ULN + bilirubin $>1$ ULN; ALT $>3 U L N$; ALT $>3$ ULN + bilirubin $>2$ ULN; ALT $>10$ ULN) and (II) algorithms based on solely liver enzyme values (ALT $>3$ ULN + bilirubin $>$ 2ULN; ALT $>$ 10ULN). Hospital medical records were reviewed to confirm final diagnosis. The positive predictive value (PPV) of each algorithm was calculated.

Results A total of 707 cases of ALI were identified. After medical review 194 (27\%) patients had confirmed idiopathic ALI. The PPV for (I) algorithms with an ICD-9-CM code as well as abnormal tests ranged from 32\% (13/41) to 48\% (43/90) with the highest PPV found with ALT $>2$ ULN. The PPV for (II) algorithms with liver test abnormalities was maximally $26 \%$ (150/571).

Conclusions The algorithm based on ICD-9-CM codes indicative of ALI combined with abnormal liver-related laboratory tests is the most efficient algorithm for identifying idiopathic ALI cases. However, cases were missed using this algorithm, because not all ALI cases had been assigned the relevant diagnostic codes in daily practice. Copyright (C) 2015 John Wiley \& Sons, Ltd.
\end{abstract}

KEY WORDS - acute liver injury; ICD-9-CM codes; laboratory measurements; validity; pharmacoepidemiology

Received 8 October 2014; Revised 27 May 2015; Accepted 28 May 2015

\section{INTRODUCTION}

Drug-induced liver injury (DILI) is a potentially serious adverse reaction and an important public health problem. It is the most common cause of postmarketing warnings and market withdrawals because of safety reasons. ${ }^{1-3}$ DILI often becomes apparent after a drug has entered the market, and there is a need

*Correspondence to: M. L. De Bruin, Department of Pharmacoepidemiology and Clinical Pharmacology, Utrecht Institute for Pharmaceutical Sciences, PO-box 80082, 3508 TB Utrecht, The Netherlands. E-mail: m.1.debruin@uu.nl to quantify risks throughout the whole product life cycle. In the post-marketing setting, observational studies are often involved when DILI is pointed out as a potential risk in the risk management plan. Because of differences in outcome definitions, selection bias or underreporting ${ }^{4}$, the incidence and risk of DILI vary among epidemiological studies. ${ }^{5-7}$ There is a need for outcome definitions of relevant endpoints in studies assessing DILI.

A method to quantify DILI risk is to compare the incidence of acute liver injury (ALI) of users of a certain drug, to a reference population. In order to avoid biases and distortion of relative risks by other causes, 
case-control studies are often restricted to idiopathic ALI cases. ${ }^{8}$ In pharmacoepidemiological studies using electronic health care record databases defining relevant endpoints for idiopathic ALI can be a challenge. Search algorithms are more and more used for this purpose and can be highly effective in detecting patients. ${ }^{9-11}$ Such algorithms can be based on diagnostic codes which are generally recorded at hospital discharge using the International Classification of Diseases (ICD). Accuracy and completeness of the diagnostic codes within databases are crucial but depend on multiple factors, such as coding quality and information in the patient records. ${ }^{12}$ Limited information is available on the utility of diagnostic codes to identify patients with idiopathic ALI. Another strategy to detect these patients is by using liver-related laboratory results on serum alanine aminotransferase (ALT), aspartate aminotransferase (AST), alkaline phosphatase (AP) and bilirubin. Different cut-off levels and combinations of these serum markers have been defined for ALI by various international expert groups. ${ }^{13,14}$ In this study we evaluated several algorithms based on different laboratory definitions and also on diagnostic codes. An algorithm that is able to accurately identify patients with idiopathic ALI would facilitate epidemiological studies to estimate the impact of medication use on the risk of developing DILI.

The aim of this study is to determine the ability of algorithms based on liver-related laboratory measurements with or without combined diagnostic codes to correctly predict idiopathic ALI cases. To address this issue, we validated the identified cases by medical record review and determined the positive predictive value (PPV).

\section{METHODS}

\section{Study design and data source}

A cross-sectional study was conducted among in- and outpatients of the University Medical Center Utrecht (UMC Utrecht). Data for this study were obtained from the Utrecht Patient Oriented Database (UPOD). UPOD comprises information on patient demographics, hospital discharge diagnoses, medical procedures, medication orders and laboratory tests for all patients treated at the UMC Utrecht. The UMC Utrecht is a 1042-bed academic medical center located in the center of the Netherlands. Approximately 165000 patients are treated annually during more than 28000 clinical hospitalizations, 15000 day-care treatments and 333000 outpatient visits. The UPOD database is described in detail elsewhere. ${ }^{15}$ Medical Review Board approval for this research project was obtained at the UMC Utrecht.

\section{Study population}

Patients aged 18 years or older, hospitalized or referred to the UMC Utrecht between 1 January 2008 and 31 December 2010 were screened for idiopathic acute liver injury in UPOD.

To identify patients with ALI two algorithms were used. Algorithm I identified cases based on diagnostic codes combined with laboratory results related to liver injury. In algorithm II, identification of potential cases of ALI was solely based on abnormal liver-related laboratory measurements.

\section{Algorithm I}

Patients with a primary or secondary International Classification of Diseases, $9^{\text {th }}$ revision, Clinical Modification (ICD-9-CM) code indicative of idiopathic acute liver injury were identified. These included hospital discharge codes for liver disease or codes referring to procedures or symptoms of liver injury (codes listed in Table 1). Further, selected patients had to meet one of the following criteria of abnormal liverrelated laboratory tests within 3 months before or after

Table 1. Selected ICD-9-CM codes and frequency of patients identified by algorithm I and number of confirmed cases after medical review

\begin{tabular}{|c|c|c|c|}
\hline $\begin{array}{l}\text { ICD- } \\
9-\mathrm{CM} \\
\text { code }\end{array}$ & Code description & $\begin{array}{l}\text { No. of } \\
\text { identified } \\
\text { ALI cases } \\
\mathrm{n}=98\end{array}$ & $\begin{array}{c}\text { No. of } \\
\text { confirmed } \\
\text { ALI cases } \\
\mathrm{n}=46\end{array}$ \\
\hline & Diagnostic codes & & \\
\hline 277.4 & Jaundice, idiopathic & - & - \\
\hline 570.0 & Acute hepatic failure & 2 & 0 \\
\hline 572.2 & Hepatic coma & 9 & 0 \\
\hline 572.4 & Hepatorenal syndrome & 3 & $1(33 \%)$ \\
\hline 572.8 & $\begin{array}{l}\text { Liver failure, not otherwise } \\
\text { specified }\end{array}$ & - & - \\
\hline 573.3 & $\begin{array}{l}\text { Hepatitis, toxic } \\
\text { (noninfectious) hepatitis }\end{array}$ & 5 & $4(80 \%)$ \\
\hline 573.8 & Jaundice, hepatocellular & - & - \\
\hline 573.9 & Unspecified disorder of liver & 73 & $41(56 \%)$ \\
\hline 782.4 & Jaundice, unspecified & 4 & 0 \\
\hline 789.1 & Hepatomegaly & 1 & 0 \\
\hline 789.59 & Other ascites & - & - \\
\hline 790.4 & $\begin{array}{l}\text { Nonspecific elevation of } \\
\text { levels of transaminase or } \\
\text { lactic acid dehydrogenase } \\
{[\text { LDH] }}\end{array}$ & - & - \\
\hline 790.5 & $\begin{array}{l}\text { Other nonspecific abnormal } \\
\text { serum enzyme levels }\end{array}$ & 1 & 0 \\
\hline 791.4 & Biliuria & - & - \\
\hline 794.8 & $\begin{array}{l}\text { Abnormal results of liver } \\
\text { function, abnormal liver scan } \\
\text { Procedure codes }\end{array}$ & - & - \\
\hline 50.1 & $\begin{array}{l}\text { Diagnostic procedures on } \\
\text { liver }\end{array}$ & - & - \\
\hline 50.5 & Liver transplant & - & - \\
\hline 50.91 & $\begin{array}{l}\text { Percutaneous aspiration of } \\
\text { liver }\end{array}$ & - & - \\
\hline
\end{tabular}


the date of assigned ICD-9-CM code: (a) an increase of more than 2 times the upper limit of the normal range (ULN) in the serum concentration of ALT (ALT > 2ULN) or (b) a combined increase in AST, AP and total bilirubin provided one of them is twice the upper limit of the respective normal range (AST $>1$ ULN $+\mathrm{AP}>1 \mathrm{ULN}+$ bilirubin $>1 \mathrm{ULN}$ ) or (c) an increase of ALT $>3$ ULN or (d) an increase of ALT $>10$ ULN or (e) ALT $>3$ ULN and bilirubin $>2$ ULN and absence of AP elevation, also referred as Hy's law criteria. ${ }^{16}$

The thresholds for ALI in algorithm Ia and $\mathrm{Ib}$ were based on the CIOMS (Council for International Organizations of Medical Sciences) laboratory criteria. ${ }^{13}$ To determine the ability of algorithms with slightly and notably higher threshold levels of ALT to correctly identify ALI cases we also included cutoff levels of ALT > 3ULN and ALT > 10ULN.

\section{Algorithm II}

Patients with the following abnormal laboratory measurements were identified: (a) an increase of ALT $>10$ ULN or (b) ALT $>3$ ULN and bilirubin2ULN and absence of AP elevation (Hy's law). Selection took place regardless the presence of diagnostic codes.

In both algorithms patients were excluded if they also had a recorded diagnostic code indicating a known other cause of liver injury (viral hepatitis, liver cancer, gallbladder disease, pancreatic disease and chronic liver disease (ICD-9-CM 070, 155, 197.7, 571.4, 571.8, 571.9, 574, 575, 576 and 577)) within 1 year before or 2 months after the acute liver injury code was assigned (algorithm I) or date of the abnormal liver-related laboratory test (algorithm II).

The medical records of all patients that were identified with algorithm I and II were manually reviewed to validate cases. A stepwise approach was used in classifying cases. ALI cases were assessed as nonidiopathic when a cause could be identified (including hepatic cancer/metastases, alcohol related, pancreaticobiliary causes, chronic liver disease, viral hepatitis, hemodynamic causes, pregnancy related and other). The diagnostic codes recorded for these cases were evaluated to identify possible other causes of liver injury. The remaining cases were classified as idiopathic. After medical review these cases were further divided into three categories (idiopathic, not ascertainable because of incomplete medical evaluation and associated with drug exposure). When all alternative causes were tested negative or the liver injury was believed idiopathic by the physician the case was classified as idiopathic. The cause was considered not ascertainable if medical evaluation for alternative causes was not complete (e.g. no virology investigations or no imaging to detect biliary obstruction was performed) and the cause could therefore not be determined. Classification as 'associated with drug exposure' was based on evaluation of the following items according to international consensus ${ }^{17}$ : information on the time to onset, the course of the reaction, risk factors, re-exposure to the drugs and previous report in the literature of cases of hepatotoxicity associated with the drug and the summary of product information (SPC).

\section{Statistical analyses}

The PPV of each algorithm for ALI was calculated as the number of idiopathic ALI cases confirmed by medical review divided by the total number of ALI cases identified by the algorithm. To provide insight in the uncertainty of the estimates the $95 \%$ confidence intervals $(\mathrm{CI})$ of the PPV were calculated.

\section{RESULTS}

\section{Algorithm I}

Between 2008 and 2010, there were 60855 patients (inpatients as well as outpatients) with one or more recorded hospital discharge diagnoses identified in the UPOD database. Of these, there were 145 patients with a diagnostic code indicative of acute liver injury. Eleven of the 145 potential cases were excluded because an alternative cause of ALI was also recorded in the database. Among the 134 remaining potential idiopathic cases identified using Algorithm I, 99 patients met one or more of the defined laboratory abnormalities related to liver injury. The medical record of 1 patient was not available. Most patients (92\%) had elevated ALT values, of which $43 \%$ had very high ALT values ( $>10 \mathrm{ULN}$, algorithm Id).

None of the identified patients had more than one ICD-9-CM code suggestive of ALI. The diagnostic code for unspecified disorder of liver (573.9) was most commonly assigned and accounted for three quarters of the assigned diagnostic codes (Table 1). Figure 1 shows a flow chart illustrating the derivation of patients with ICD-9-CM codes in combination with liver-related laboratory measurements.

\section{Algorithm II}

During the study period, a liver-related laboratory test was performed for 61841 patients. Among the 798 patients with laboratory results indicating liver injury 


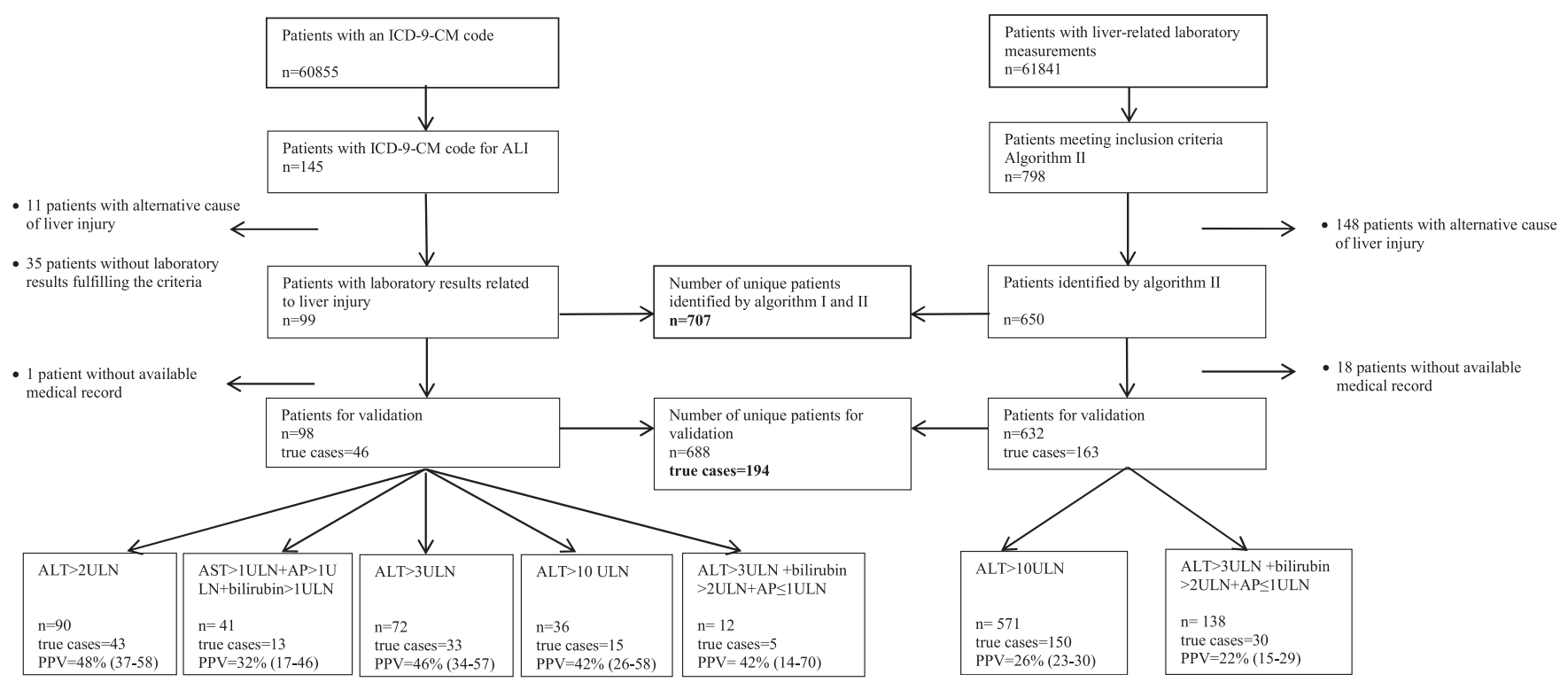

Figure 1. Flow chart of algorithm I and II: number of patients identified with liver injury diagnostic codes and liver-related laboratory abnormalities and number of true cases, with positive predictive values (PPV) (95\% confidence interval)

according to algorithm II, 148 patients were excluded because an ICD-9-CM code indicating an alternative cause of liver injury had been assigned (Figure 1). Of 18 patients medical records were not available. Most of the 632 remaining patients fulfilled the criteria of ALT $>10$ ULN (90\%, algorithm IIa) and a small proportion had laboratory measurements according to Hy's law criteria (22\%, algorithm IIb). An overlap of 77 patients existed between algorithm IIa and IIb.

Using algorithms I and II combined, a total of 707 potential cases were identified. Between the algorithms there was an overlap of 42 patients, of which 15 individuals were true idiopathic ALI cases. The electronic medical records were available for 688 patients $(97 \%)$. After medical record review, idiopathic ALI was confirmed in $28 \%$ of these patients (194/ 688). Algorithm I provided 46 true idiopathic ALI cases of the 98 identified potential cases (47\%), whereas the number of true cases in algorithm II was 163 out of the 632 potential cases $(26 \%)$. Table 2 shows the causes of liver injury and corresponding number of patients and percentages per algorithm and subcategory. In bold are the overall numbers specified of idiopathic causes with the PPV and 95\% CI. In both algorithms were more non-idiopathic causes identified for ALI than idiopathic. Hemodynamic causes accounted for the highest proportion of the non-idiopathic causes in both algorithms. In addition, in algorithm I alcohol related causes of ALI could be seen in the categories with AST and elevated bilirubin. In algorithm II also pancreaticobiliary causes and viral hepatitis were frequently identified as alternative causes. The most assigned hospital diagnostic codes for the identified non-idiopathic ALI cases appeared ICD code 427, 799, E878 and 428 (respectively, cardiac dysrhythmias, other ill-defined conditions, surgical operation and procedure, and heart failure). Of the idiopathic causes, a large proportion of ALI cases was associated with drug use in algorithm I and to a lesser extent also in algorithm II. In fact, drug associated liver injury was the most identified cause in all categories in algorithm I.

The PPVs for idiopathic ALI of the laboratory measurements ALT $>10 \mathrm{ULN}$ and ALT $>3 \mathrm{ULN}+$ bilirubin $>2$ ULN (Hy's law) of algorithm II were $26 \%$ (150/571) and 22\% (30/138) respectively. Abnormal liver-related laboratory tests in combination with an ICD-9-CM code increased PPV and ranged from $32 \%(13 / 41)$ for a combined increase in AST, AP and bilirubin (algorithm $\mathrm{Ib}$ ) to $48 \%$ (43/90) for ALT $>2$ ULN.

\section{DISCUSSION}

This study evaluated the ability of algorithms based on hospital discharge diagnoses and laboratory measurements to identify idiopathic ALI cases in a hospitalbased electronic health care record database. The algorithms based solely on abnormal liver-related laboratory tests, algorithm IIa and IIb together, provided the largest absolute number of true idiopathic ALI cases $(n=163)$. However, $74 \%$ of the initially 
Table 2. Frequencies and percentages of causes of acute liver injury per algorithm and category

\begin{tabular}{|c|c|c|c|c|c|c|c|}
\hline \multirow{3}{*}{ Cause ALI } & \multicolumn{5}{|c|}{ Algorithm I (ICD-9-CM codes + liver-related enzyme values) } & \multicolumn{2}{|c|}{$\begin{array}{l}\text { Algorithm II (liver-related enzyme } \\
\text { values only) }\end{array}$} \\
\hline & ALT $>2$ ULN & $\begin{array}{c}\mathrm{AST}>1 \mathrm{ULN}+ \\
\mathrm{AP}>1 \mathrm{ULN}+ \\
\mathrm{BIL}>1 \mathrm{ULN}\end{array}$ & ALT $>3$ ULN & ALT $>10$ ULN & $\begin{array}{c}\mathrm{ALT}>3 \mathrm{ULN}+ \\
\mathrm{BIL}>2 \mathrm{ULN}+ \\
\mathrm{AP} \leq 1 \mathrm{ULN}\end{array}$ & ALT $>10$ ULN & $\begin{array}{c}\text { ALT }>3 \text { ULN + } \\
\text { BIL }>2 \text { ULN + } \\
\text { AP } \leq 1 \text { ULN }\end{array}$ \\
\hline & $\mathrm{n}=90$ & $\mathrm{n}=41$ & $\mathrm{n}=72$ & $\mathrm{n}=36$ & $\mathrm{n}=12$ & $\mathrm{n}=571$ & $\mathrm{n}=138$ \\
\hline \multicolumn{8}{|l|}{$\begin{array}{l}\text { Idiopathic and drug- } \\
\text { associated }\end{array}$} \\
\hline $\begin{array}{l}n(\%) \\
(95 \% C I)\end{array}$ & $\begin{array}{c}43(48 \%) \\
\quad(37-58)\end{array}$ & $13(32 \%)$ & $33(46 \%)$ & $15(42 \%)$ & $5(42 \%)$ & $150(26 \%)$ & $30(22 \%)$ \\
\hline Idiopathic & $2(2 \%)$ & $0(0 \%)$ & $1(1 \%)$ & $0(0 \%)$ & $0(0 \%)$ & $5(1 \%)$ & $0(0 \%)$ \\
\hline $\begin{array}{l}\text { Not ascertainable } \\
\text { because of incomplete } \\
\text { medical evaluation }\end{array}$ & $14(16 \%)$ & $3(7 \%)$ & $11(15 \%)$ & $4(11 \%)$ & $0(0 \%)$ & $63(11 \%)$ & $13(9 \%)$ \\
\hline Drug-associated & $27(30 \%)$ & $10(24 \%)$ & $21(30 \%)$ & $11(31 \%)$ & $5(42 \%)$ & $82(14 \%)$ & $17(12 \%)$ \\
\hline Non-idiopathic & $47(52 \%)$ & $28(68 \%)$ & $39(54 \%)$ & $21(58 \%)$ & $7(58 \%)$ & $421(74 \%)$ & $108(78 \%)$ \\
\hline $\begin{array}{l}\text { Liver cancer or } \\
\text { metastases }\end{array}$ & $2(2 \%)$ & $1(2 \%)$ & $2(3 \%)$ & $1(3 \%)$ & $0(0 \%)$ & $18(3 \%)$ & $5(4 \%)$ \\
\hline Alcohol related & $3(3 \%)$ & $5(12 \%)$ & $3(4 \%)$ & $1(3 \%)$ & $3(25 \%)$ & $3(1 \%)$ & $7(5 \%)$ \\
\hline $\begin{array}{l}\text { Pancreaticobiliairy } \\
\text { causes }\end{array}$ & $6(7 \%)$ & $4(10 \%)$ & $5(7 \%)$ & $3(8 \%)$ & $0(0 \%)$ & $70(12 \%)$ & $9(7 \%)$ \\
\hline Chronic liver disease & $5(6 \%)$ & $3(7 \%)$ & $2(3 \%)$ & $0(0 \%)$ & $1(8 \%)$ & $13(2 \%)$ & $7(5 \%)$ \\
\hline Viral hepatitis & $5(6 \%)$ & $2(5 \%)$ & $4(6 \%)$ & $3(8 \%)$ & $0(0 \%)$ & $43(8 \%)$ & $13(9 \%)$ \\
\hline Hemodynamic causes & $12(13 \%)$ & $7(17 \%)$ & $12(17 \%)$ & $7(19 \%)$ & $2(17 \%)$ & $156(27 \%)$ & $40(29 \%)$ \\
\hline Pregnancy related & $0(0 \%)$ & $0(0 \%)$ & $0(0 \%)$ & $0(0 \%)$ & $0(0 \%)$ & $21(4 \%)$ & $1(1 \%)$ \\
\hline $\begin{array}{l}\text { Graft versus Host } \\
\text { disease }\end{array}$ & $1(1 \%)$ & $0(0 \%)$ & $1(1 \%)$ & $1(3 \%)$ & $0(0 \%)$ & $22(4 \%)$ & $6(4 \%)$ \\
\hline Other & $13(14 \%)$ & $6(15 \%)$ & $10(14 \%)$ & $5(14 \%)$ & $1(8 \%)$ & $75(13 \%)$ & $20(15 \%)$ \\
\hline
\end{tabular}

$\mathrm{ALI}=$ acute liver injury; ICD-9-CM $=$ International Classification of Diseases, $9^{\text {th }}$ revision, Clinical Modification; $95 \% \mathrm{CI}=95 \%$ confidence interval; $\mathrm{ALT}=$ alanine aminotransferase; $\mathrm{AST}=$ aspartate aminotransferase; $\mathrm{AP}=$ alkaline phosphatase; $\mathrm{BIL}=$ bilirubin; $\mathrm{ULN}=$ upper limit of normal.

identified cases in these algorithms had alternative causes and were false positive. For the algorithms using a combination of ICD-9-CM codes and laboratory measurements (algorithm I) the ability to correctly identify idiopathic ALI cases was improved. Although the latter algorithms are therefore the most efficient search strategy, positive predictive values remained low in all subcategories of the algorithm (below $50 \%$ ). This means that if such algorithms are used in observational studies for case identification without further validation a possible association might be distorted.

Furthermore, our results showed that although the search strategies based on ICD-9-CM codes combined with laboratory measurements had highest PPV scores, these algorithms were not very sensitive. The majority of true idiopathic ALI cases provided by liver-related laboratory abnormalities only (algorithm II), were not captured by algorithm I (91\%). This indicates that many ALI cases were not coded as such in the health care record database. This has serious implications for epidemiological research, as the inability to identify most ALI cases may lead to underestimations of for instance absolute incidence numbers in cohort studies. In addition, for use in case finding this algorithm is also not very efficient as the absolute numbers of cases were low.

Our study showed that even modest elevated ALT values (an increase of more than $2 \mathrm{ULN}$ ) can represent idiopathic ALI cases. This threshold is one of the two laboratory criteria for liver injury according to the CIOMS. Of the 18 cases with serum ALT $>2$ ULN which were not present in the higher ALT categories of algorithm I, there were 10 true idiopathic cases. This ALT level combined with diagnostic codes is able to detect less severe ALI and is equally capable in predicting true idiopathic cases as higher levels of ALT. This demonstrates that it is difficult to differentiate between etiologies based on ALT values. A combined increase in AST, AP and bilirubin, with one of these more than two times ULN (the second criterion defined by CIOMS), had the lowest predictability of algorithm I.

Imprecise coding of non-idiopathic ALI appeared to be the reason for the generally low PPVs in both algorithms. This may reflect the sometimes extended process of determining the cause of the liver injury what makes that initially a nonspecific liver injury code was chosen for the elevated laboratory results. It may also have been because of inexact coding by the 
coding staff or to inaccurate information written by the physician. A proportion of the cases had liver disorders because of a cause that was not captured in the diagnostic codes for exclusion, such as hemodynamic causes like cardiomyopathy or shock. It may be worthwhile to include ICD codes reflecting these conditions (e.g. 427.5 cardiac arrest or E878 codes for surgical operation and procedures) in hospital-based data for exclusion purposes. A reason for the fact that the liver injury was not coded at all could be that some conditions are considered less important than others in patients with multiple comorbidities or seen as part of the whole clinical spectrum of the patient and therefore were not recorded.

Few studies have evaluated the validity of ICD codes to identify idiopathic ALI cases. Lo Re et al. selected several ICD-9-CM codes indicative of severe acute liver injury and acute liver failure and found a PPV of $25 \%$ (26/105). ${ }^{18}$ Combinations of diagnostic codes resulted in higher PPV for these outcomes. Diagnostic codes 570 together with 572.8 had a PPV of $100 \%$ and code 570 in combination with 572.2 a PPV of $67 \%$. However, the number of identified cases was small ( 7 and 3 , respectively). Moreover, this finding may also reflect considerable heterogeneity in coding conventions as in our study only single diagnostic codes indicative of idiopathic ALI were assigned. The diagnostic code 572.8 (other sequelae of chronic liver diseases) was not identified in our database, and none of the cases with diagnostic code 572.2 was a true idiopathic ALI case. In our study the code 573.3 had the highest PPV (80\%), although only 5 cases were identified. In another study a PPV of $2 \%$ (119/7395) was found, in which ICD-9$\mathrm{CM}$ codes were used to identify cases of drug-induced liver injury because of four selected drugs. ${ }^{19}$ In this latter study patients with a recorded diagnostic code suggesting other causes for the liver injury were not excluded before validation, which might in part explain the very low PPV compared to our results.

Also laboratory abnormalities associated with liver injury have been used in studies to identify cases of DILI. These studies show results in line with ours in that low predictive values were found. Xu et al. identified 129 hospitalized patients with ALT $>10$ ULN and DILI accounted for $19 \%$ of cases. ${ }^{20}$ In a prospective study hospitalized patients were selected based on ALT $>2$ ULN and AP $>1.5 \mathrm{ULN}$ if associated with an increase of ALT or gamma glutamyl transpeptidase or bilirubin, 13 cases of DILI were validated of the 147 identified cases (9\%). ${ }^{21}$ In another study among 128 patients with ALT $>3$ ULN 7 cases were validated with DILI (5\%). ${ }^{22}$ In a study identifying ALI cases in primary care databases in Spain and the United
Kingdom using the same restrictive algorithm including both diagnostic codes and laboratory measurements, PPVs of, respectively, 24\% (43/179) and 63\% (64/101) were found. ${ }^{23}$

These results highlight the difficulty of identifying idiopathic ALI cases in electronic health care record databases and the need for additional ways to capture cases. It might be valuable to set up a registry which includes prospectively idiopathic ALI cases. An example of such a registry, albeit for a different clinical outcome, is the Amsterdam Resuscitation Studies, in which data from cases of cardiac arrest in the Netherlands are collected and stored in the ARREST database. ${ }^{24}$ This database was specifically designed to investigate the determinants and outcomes of cardiac arrest and proved to be suitable for epidemiological research on druginduced sudden cardiac arrest. ${ }^{25}$

The strength of this study is that two different algorithms of case identification were evaluated. This way we were able to compare two strategies as it provided data on predictability and relative sensitivity. Furthermore, we used several thresholds of liver related laboratory levels to estimate the ability of identifying cases.

A limitation of this study is that it was conducted in one hospital, and therefore the results might not be generalizable to other hospitals. Variable regimens in assigning diagnostic codes might generate other results. Different health care systems and health-seeking behavior can also be of influence. ${ }^{6}$ For the present study we used ALT, AST, AP and bilirubin values to capture liver function. It may be worthwhile to study the added value of incorporating gamma glutamyl transpeptidase (gamma GT) in lab-based algorithms in future validation studies. Another limitation is that the sensitivity of the algorithms could not be estimated, because we did not evaluate idiopathic ALI among the patients that were not identified by the algorithms. Therefore, we did not know the number of true cases. However, comparing the two algorithms, the sensitivity of algorithm I was low as only a small proportion of the true cases identified by algorithm II was detected (9\%). Algorithm II detected $33 \%$ of the true cases identified by algorithm I.

In conclusion, algorithms to identify idiopathic ALI based on liver-related laboratory measurements, even in combination with hospital discharge diagnoses, are not very suitable for selecting cases in electronic health care record databases. Manual review of medical records is deemed necessary to validate cases. Algorithm based on ICD-9-CM codes indicative of ALI combined with abnormal liver-related laboratory tests are the most efficient. However, cases were missed using this algorithm, because not all ALI cases have been assigned the relevant diagnostic codes in daily practice. 
This study highlights the challenges encountered when electronic health care record databases are used for research on ALI associated with drugs. Other ways of case finding may be warranted. Surveillance registries that include cases of idiopathic ALI could be of value for future research on DILI.

\section{CONFLICT OF INTEREST}

The research leading to these results was conducted as part of the PROTECT consortium (Pharmacoepidemiology Research on Outcomes of Therapeutics by a European ConsorTium, www.imi-protect.eu) which is a public-private partnership coordinated by the European Medicines Agency. The Department of Pharmacoepidemiology and Clinical Pharmacology, Utrecht Institute for Pharmaceutical Sciences, Utrecht University has received unrestricted research funding from the Netherlands Organisation for Health Research and Development (ZonMW), the Dutch Health Care Insurance Board (CVZ), the Royal Dutch Pharmacists Association (KNMP), the private-public funded Top Institute Pharma (www.tipharma.nl, includes co-funding from universities, government and industry), the EU Innovative Medicines Initiative (IMI), EU 7th Framework Program (FP7), the Dutch Medicines Evaluation Board and the Dutch Ministry of Health and Industry (including GlaxoSmithKline, Pfizer, and others).

\section{KEY POINTS}

- Search algorithms to identify idiopathic ALI cases applied in a Dutch hospital-based health care database appear not to be accurate.

- Algorithms based on abnormal laboratory measurements indicating liver injury show low PVVs (22\% and 26\%), mainly because of inclusion of false positive cases with alternative causes of ALI.

- A search strategy combining laboratory measurements and diagnostic ICD-9-CM codes indicative of liver injury improves the ability of detecting true cases with maximum PPV of $48 \%$. However, ALI patients are often not assigned a corresponding diagnostic code and are therefore missed by this algorithm.

- For case finding purposes, algorithms based on liver-related laboratory measurements might be considered as this search strategy yields the largest number of true cases after medical review.

\section{ETHICS STATEMENT}

Approval for this study was obtained by the Institutional Review Board of UMC Utrecht.

\section{ACKNOWLEDGEMENTS}

The PROTECT project has received support from the Innovative Medicine Initiative Joint Undertaking (www.imi.europa.eu) under Grant Agreement no 115004 , resources of which are composed of financial contribution from the European Union's Seventh Framework Programme (FP7/2007-2013) and EFPIA companies' in kind contribution. The views expressed are those of the authors only and not of their respective institution or company.

\section{REFERENCES}

1. Bakke OM, Manocchia M, de Abajo F, Kaitin KI, Lasagna L. Drug safety discontinuations in the United Kingdom, the United States, and Spain from 1974 through 1993: a regulatory perspective. Clin Pharmacol Ther 1995; 58(1): 108-117.

2. Kaplowitz N. Idiosyncratic drug hepatotoxicity. Nat Rev Drug Discov 2005; 4(6): 489-499.

3. Lasser KE, Allen PD, Woolhandler SJ, Himmelstein DU, Wolfe SM, Bor DH. Timing of new black box warnings and withdrawals for prescription medications. JAMA 2002; 287(17): 2215-2220.

4. Verma S, Kaplowitz N. Diagnosis, management and prevention of drug-induced liver injury. Gut 2009; 58(11): 1555-1564. doi:10.1136/gut.2008.163675.

5. Bjornsson ES, Bergmann OM, Bjornsson HK, Kvaran RB, Olafsson S. Incidence, presentation, and outcomes in patients with drug-induced liver injury in the general population of Iceland. Gastroenterology 2013; 144(7): 1419-1425.e3.

6. Ferrajolo C, Verhamme KM, Trifiro G, et al. Idiopathic acute liver injury in paediatric outpatients: Incidence and signal detection in two European countries. Drug Saf 2013; 36(10): 1007-1016.

7. Sgro C, Clinard F, Ouazir K, et al. Incidence of drug-induced hepatic injuries: a French population-based study. Hepatology 2002; 36(2): 451-455.

8. Jick H, Vessey MP. Case-control studies in the evaluation of drug-induced illness. Am J Epidemiol 1978; 107(1): 1-7.

9. Hanatani T, Sai K, Tohkin M, et al. An algorithm for the identification of heparin-induced thrombocytopenia using a medical information database. J Clin Pharm Ther 2013; 38(5): 423-428.

10. Sai K, Hanatani T, Azuma Y, et al. Development of a detection algorithm for statin-induced myopathy using electronic medical records. J Clin Pharm Ther $2013 ; \mathbf{3 8}(3)$ : 230-235.

11. ten Berg MJ, van Solinge WW, van den Bemt PM, Huisman A, Schobben AF, Egberts TC. Platelet measurements versus discharge diagnoses for identification of patients with potential drug-induced thrombocytopenia: a cross-sectional study in the Netherlands. Drug Saf 2009; 32(1): 69-76.

12. Benchimol EI, Manuel DG, To T, Griffiths AM, Rabeneck L, Guttmann A. Development and use of reporting guidelines for assessing the quality of validation studies of health administrative data. J Clin Epidemiol 2011; 64(8): 821-829.

13. Benichou C. Criteria of drug-induced liver disorders. report of an international consensus meeting. J Hepatol 1990; 11(2): 272-276.

14. Aithal GP, Watkins PB, Andrade RJ, et al. Case definition and phenotype standardization in drug-induced liver injury. Clin Pharmacol Ther 2011; 89(6): 806-815. doi:10.1038/clpt.2011.58.

15. ten Berg MJ, Huisman A, van den Bemt PM, Schobben AF, Egberts AC, van Solinge WW. Linking laboratory and medication data: new opportunities for pharmacoepidemiological research. Clin Chem Lab Med 2007; 45(1): 13-19.

16. Temple R. Hy's law: predicting serious hepatotoxicity. Pharmacoepidemiol Drug Saf 2006; 15(4): 241-243.

17. Danan G, Benichou C. Causality assessment of adverse reactions to drugs-I. A novel method based on the conclusions of international consensus meetings: application to drug-induced liver injuries. J Clin Epidemiol 1993; 46(11): 1323-1330.

18. Lo Re V, 3rd, Haynes K, Goldberg D, et al. Validity of diagnostic codes to identify cases of severe acute liver injury in the U.S. food and drug administration's 
mini-sentinel distributed database. Pharmacoepidemiol Drug Saf 2013; 22(8): $861-872$.

19. Jinjuvadia K, Kwan W, Fontana RJ. Searching for a needle in a haystack: use of ICD-9-CM codes in drug-induced liver injury. Am J Gastroenterol 2007; 102(11): 2437-2443. doi:10.1111/j.1572-0241.2007.01456.x.

20. Xu HM, Chen Y, Xu J, Zhou Q. Drug-induced liver injury in hospitalized patients with notably elevated alanine aminotransferase. World J Gastroenterol 2012; 18(41): 5972-5978.

21. Bagheri H, Michel F, Lapeyre-Mestre M, et al. Detection and incidence of druginduced liver injuries in hospital: a prospective analysis from laboratory signals. Br J Clin Pharmacol 2000; 50(5): 479-484.
22. M'Kada H, Perazzo H, Munteanu M, et al. Real time identification of druginduced liver injury (DILI) through daily screening of ALT results: a prospective pilot cohort study. PLoS One 2012; 7(8): e42418.

23. Ruigómez A, Brauer R, García Rodríguez L, et al. Ascertainment of acute liver injury in two european primary care databases. Eur J Clin Pharmacol 2014; 70(10): 1227-1235.

24. Bardai A, Berdowski J, van der Werf C, et al. Incidence, causes, and outcomes of outof-hospital cardiac arrest in children. A comprehensive, prospective, population-based study in the Netherlands. J Am Coll Cardiol 2011; 57(18): 1822-1828.

25. Warnier MJ, Blom MT, Bardai A, et al. Increased risk of sudden cardiac arrest in obstructive pulmonary disease: a case-control study. PLoS One 2013; 8(6): e65638. 